\title{
Enhancing User-Engagement in the Design Process through Augmented Reality Applications
}

\author{
Catherine Erzeticl ${ }^{\text {, Tiara Dobbs }}{ }^{2}$, Alessandra Fabbri ${ }^{3}$, \\ Nicole Gardner ${ }^{4}$, M. Hank Haeusler ${ }^{5}$, Yannis Zavoleas ${ }^{6}$ \\ 1,3,4 UNSW / Computational Design ${ }^{2} P T W$ Sydney ${ }^{5}$ UNSW / Computational Design \\ \& CAFA Visual Innovation Institute Beijing ${ }^{6} U N S W /$ Computational Design \\ 1,3,4,5,6 \{c.erzetic|a.fabbri|n.gardner|m.haeusler|y.zavoleas\}@unsw.edu. \\ au²Tiara.Dobbs@ptw.com.au
}

\begin{abstract}
Augmented Reality (AR) technologies are often perceived as the most impactful method to enhance the communication between the designer and the client during the iterative design process. However, the significance of designing the User Interface (UI) and the User Experience (UX) are often underestimated. To intercede, this research aims to employ new and existing techniques to develop UI's, and comparatively assess "the accuracy and completeness with which specified users can achieve specified goals in particular environments" (Stone, 2005) - a notion this research delineates as 'effectiveness'. Prompted by the work of key scholars, the developed UI's were assessed through the lens of existing UI evaluation techniques, including: Usability Heuristics (Nielsen, 1994) and Visual and Cognitive Heuristics (Zuk and Carpendale, 2006). In partnership with PTW Architects, characteristics such as the rapidity and complexity of interactions, in conjunction with the interface's simplicity and intuitiveness, were extracted from 15 trials underwent by architectural practitioners. The outcomes of this research highlights strategies for the effective development of user interface design for mobile augmented reality applications.
\end{abstract}

Keywords: User Interface, Human Centered Design, User Experience, Heuristics, Usability Inspection Method

\section{INTRODUCTION}

Architectural practices often adopt digital applications as a means to showcase design iterations to their clients. This enhances the communication between the designer and the client during the iterative design process. Existing methods include 2D and 3D representation media. However, static imagery is limited as it lacks the ability for real-time
\end{abstract}

customisation. The communication between the designer and the client can then prove troublesome when the design intent is not mutually understood. To test the 'effectiveness' - defined by Stone as "the accuracy and completeness with which specified users can achieve specified goals" (2005) - the presented research adopted Nielsen's (1994) usability inspection methodology. This involves the use of a cus- 
tomised set of heuristics, which will be leveraged to test the effectiveness of a designed UI in a Mobile Augmented Reality (MAR) application. These heuristics are an existing technique of evaluating application effectiveness following a set of criteria. This draws on key scholars who have explored existing UI evaluation techniques, and defined existing heuristics, including: Usability Heuristics (Nielsen 1994) and Visual and Cognitive Heuristics (Zuk and Carpendale 2006) with the aim to provide an "immediate validation approach" (Munzner 2009). Within this research, these concepts will be explored and evaluated for future development of the UI and UX of MAR applications.

The presented findings and this project are in collaboration with PTW Architects and is a part of a larger project scope including work published in Paneras' et al (2018) research paper Augmented Reality in the Design Process - Using visual effects (VFX) motion tracking techniques to conduct quantification research on the performance of augmented reality that explores quantification methods of motion tracking in augmented reality.

\section{RESEARCH AIMS AND QUESTIONS}

The aim of this research is to conceptualise and build various user interface layouts for visualising 3D objects in an interior design context with a project provided by PTW Architects. The UI and UX will then be tested and evaluated against project specific heuristics. The overarching expectation of this application is to enhance the design process, by streamlining the communication between the client and the designer. Therefore, this study poses the following questions:

- How effective can the application of MAR tools be in an interior design context?

-What criteria can be applied to test the effectiveness of a MAR user interface in an interior design context?

- How effective is a project specific set of heuristics at the beginning of the development process vs after user testing?

\section{METHODOLOGY}

The objective of this research is to develop an 'effective' UI for MAR applications. The developed UIs will be evaluated, providing feedback which can be leveraged to improve its design. This lends itself to an iterative development process - one that is used in an action research methodology. Thus, theoretical knowledge gained through literature review and practical prototyping were intertwined towards the realisation of effective UI options.

User testing was conducted after the development of each UI, generating both quantitative and qualitative data. A questionnaire, based on the Analytic Hierarchy Process (AHP) model (Meei Hao Hoo et al. 2013), provided the user with a rating system, capturing the quality of certain aspects of the user experience. Further, numerical data was gathered by observing the participant, revealing the number of taps and time taken to complete the set tasks. The qualitative data was collected at the end of the questionnaire to gather a comments, suggestions, and a preferred prototype. Each set of results were used to inform the creation of subsequent prototypes.

The prototypes themselves were created using a deductive methodology, whereby the research outcomes test pre-existing or generated theories. Prototype $A$ builds upon a critical review of existing MAR, or similar applications, which highlighted the limitations and successes of clarity and navigation. Prototype B was created through a set of customised usability heuristics, stemming from Nielsen (1994), in conjunction with Zuk and Carpendale's (2006) visual and cognitive heuristics, as a standard to examine the applications' interface design. Finally, to inform prototype $C$, an experiment measures prototype $A$ and prototype B's usability and visual and cognitive performance, to lead to establishing what achieves an effective application.

\section{BACKGROUND RESEARCH Critical review of existing applications}

Jakob Nielsen, inventor of prominent usability inspection methods, asserts the importance of devel- 
Table 1

Critical review of existing applications measured against customised heuristics.

Figure 1 Layered iterative design process. oping a unique set of heuristics to assess UX effectiveness, which is dependant on the interface's context. Stemming from usability heuristics of Nielsen (1994), and the visual and cognitive heuristics of Zuk and Carpendale (2006), this research tailored a customised set of heuristics for the context of interior design. Investigating existing MAR applications, this research compiled a list of 5 existing mobile applications to inform the development of prototype A: Augment, Graphisoft BIMX, MagicPlan, Ikea Catalogue and Layar. Leveraging the AHP model (Meei Hao Hoo et al. 2013), the results of the critical review of the aforementioned MARs are shown below (See Table 1).

\begin{tabular}{|c|c|c|c|c|c|c|c|c|c|c|}
\hline \multirow[t]{2}{*}{ Application } & \multicolumn{5}{|c|}{ Usability } & \multicolumn{5}{|c|}{ Visual and Cognitive } \\
\hline & 1 & 2 & 3 & 4 & 5 & 6 & 7 & 8 & 9 & 10 \\
\hline Augment & $\bullet$ & $\bullet$ & O & - & $\bullet$ & $\bullet$ & • & O & - & $\bullet$ \\
\hline $\begin{array}{l}\text { Graphisoft } \\
\text { BIMX }\end{array}$ & O & 0 & 0 & 0 & (1) & 0 & D & 0 & D & 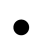 \\
\hline MagicPlan & $\bullet$ & D & O & (1) & (1) & O & D & 0 & O & (1) \\
\hline Ikea Catalogue & ○ & $\bullet$ & $\bullet$ & 0 & $\mathrm{O}$ & O & 0 & 0 & $\bullet$ & 0 \\
\hline Layer & - & $\bullet$ & 0 & 0 & 0 & - & - & 0 & - & $\bullet$ \\
\hline
\end{tabular}

\section{Tools to measure effectiveness: The Experi- ment}

To inform prototype $C$, an experiment measures prototype A and prototype B's usability and visual and cognitive performance, to lead to establishing what achieves an effective application. The following methods can be used to measure application 'effectiveness':

- Experiment: Somewhat controlled conditions and consented observation.

- Field Study: Involves observation often without consented observation.

- Surveys: Questionnaires specifically designed for a group of participants.

- Inspection methods: Heuristic evaluation is a style of inspection method to measure application performance.

- Mixed methods: Combining multiple tools.
This study engages with a mixed methods approach by combining an experiment, surveys, and heuristic evaluation as the usability inspection method.

\section{User Testing with customised Heuristics}

User-testing commenced after the completion of prototype $A$ and prototype $B$. The aim of user-testing is to evaluate the effectiveness of UI and UX design of MAR applications, through an experimental investigation. Results were predicted using the customised set of heuristics prior to user-testing, which were subsequently either validated or disproved. These experiments were developed to identify limitations and highlight user-friendly solutions.

\section{PROTOTYPING / CASE STUDY}

In collaboration with PTW Architects, three MAR UI prototypes were developed following a mixed methods approach. The iterative design process, defined in the methodology, shows the interconnecting sequential process to achieve each prototype. The initial stage involved developing a customised heuristic set (table 2) to define key objectives for both the comparison study and the development of prototype A. The prototypes were designed accordingly in Adobe Experience Design CC (Beta) 2017 and a simulated UX was achieved through the InvisionApp platform.

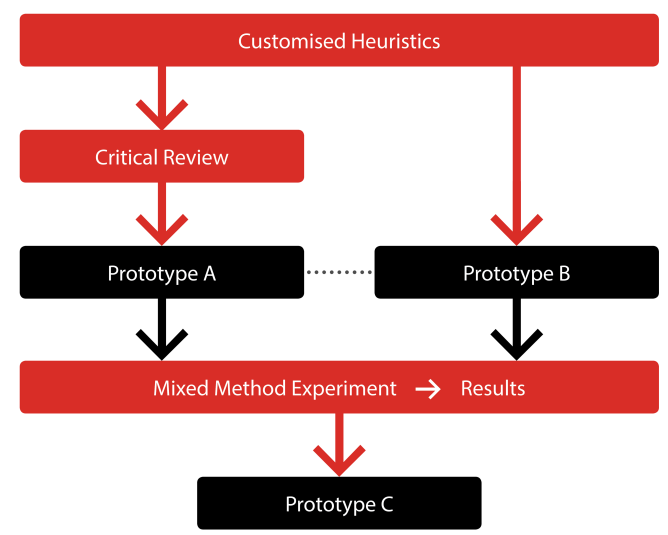




\section{Iterative Design Process}

Two types of software were required during the iterative design process; one to create the UI and the other to incorporate UX. Multiple software including Adobe Experience Design CC (Beta) 2017, Adobe Dreamweaver CC 2017, Webflow, and InvisionApp, were investigated to determine which is the most suited for the design project requirements. It was found that Adobe Experience Design CC (Beta) 2017 in combination with InvisionApp is the most accessible and time efficient prototyping tools to adhere to time constraints of the project.

UI Software. Adobe Experience Design CC (Beta) 2017 was used to iterate UI layouts for each prototype. It allowed for the exporting of PNG or JPEG file formats to be quickly generated for InvisionApp. While having the ability to assign buttons and gestures within this software, accessibility to a device with iOS $10.0+$ or android 5.0+ restricted our ability to display the UX on available tablets.

UX Software. InvisionApp was used to simulate the UX of each prototype to explore the functionality and flow of the system. The application converts PNG and JPEG to a simulated working application. It works with layers, where each screen hosts "hotspots" as buttons or gestures as triggers to redirect to other layers in the prototype, simulating a working application. To share the prototypes, the application is available to be viewed on any device through a web link.

\section{Customised Heuristics}

Heuristics 1 to 5 focus on usability performance, while 6 to 10 focus on visual and cognitive aspects (Table 2).

\section{Heuristic Rationalisation}

The development of the customised heuristics selects criteria based on their appropriateness for project objectives, as well as with constant regard for the purpose of MARUI development in an interior design context. It also provides a basis for the iterative design development process, as they can be used as a benchmark for measuring the effectiveness of MAR application prototypes.

\begin{tabular}{ll}
\hline Heuristics \\
\hline 1 & Reasonable waiting times \\
2 & Support user's concept learning \\
3 & Visual cues to influence experience \\
4 & Clear warning and exit messages \\
5 & Consistent button sizes \\
6 & Navigation simplicity \\
7 & Applies Gestalt laws \\
8 & Multiple levels of detail \\
9 & Aesthetic and minimalist design \\
10 & Designed for context \\
\hline
\end{tabular}

Table 2

Customised heuristic set.
The heuristics are justified based on their relevance to MAR UI design and the project context:

1. Although waiting times will be simulated, this is a major factor in MAR application design, as users should be able to quickly complete tasks, as it is in real-time. This heuristic may be more relevant to further work in the MAR application process.

2. Concept learning reduces time taken to complete a task in a given application, thus overtime the tool will be more efficiently used. Familiar icons support cognitive functions and enhance learnability.

3. Important for the flow of information to be accurately presented to the user. It engages the user as it reduces confusion and uncertainty while completing tasks.

4. Providing user's clarification of their actions can improve UX. Especially important in MAR applications, as users are customising their work according to their personal preferences, a sudden exit by accident can be disastrous.

5. Consistency of button size is key in achieving an aesthetically appealing UI as it presents itself as clear and straightforward.

6. How well a user can navigate through an application influences the UX. This flow can be enhanced by addressing other heuristics which can include a simplistic design and additional visual cues for example. 
Figure 2

Prototype $\mathrm{A}$

Interface
Figure 3

Prototype B Interface
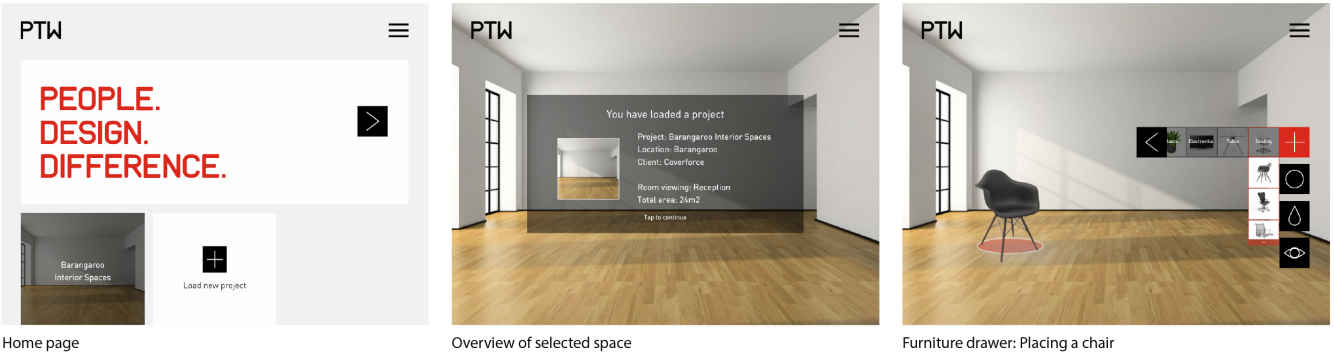

Furniture drawer: Placing a chair
7. Essential in ensuring users are able to easily navigate the controls on the MAR applications UI. Placement and order of these controls is essential in enhancing usability and visual appeal.

8. In the context of interior design, level of detail is essential as it suggests the importance of a basic and advanced interface for different experience level users.

9. To ensure users are not overwhelmed with the amount of content on any given screen, a simplistic and minimalist design is essential. It is important to present controls in an organised manner to enhance user workflow.

10. Important to include users in the iterative design process of UI and UX development as the intended demographic will provide insight to their needs and suggestions for a design tool.

In the following the three prototypes are presented and discussed (See Figure 2, Figure 3 and Figure 4).

\section{Prototype A}

The main focus was to develop a prototype that addresses the successes and limitations of the study comparison of existing applications. While also following a minimalist style to improve the UX and reduce confusion.

\section{Prototype B}

Prototype B combines functionality of prototype A, but places it in a more refined UI. It promotes the context through its aesthetically appealing interface and improved UX. A different impression is achieved in the steps of opening a project, but the "screen viewer" remains similar to that of prototype A.

\section{Prototype C}

Prototype $C$ was realised through an experiment where limitations of prototype A and B were anal-

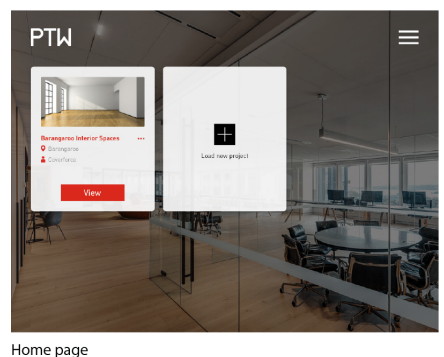

Home page

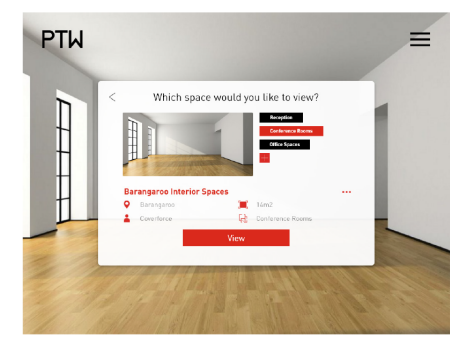

Overview of selected space

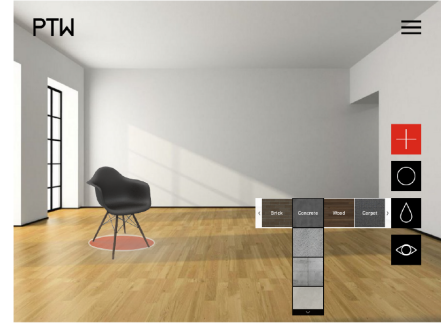

Furniture drawer: Placing a chair 
ysed and evaluated to validate improvements of prototype $C$.

\begin{tabular}{|c|c|c|}
\hline Data Type & Aims & Data Collection Method \\
\hline Quantitative & 1,4 & $\begin{array}{l}\text { Using AHP model and measuring } \\
\text { against customised heuristics. }\end{array}$ \\
\hline Quantitative & 2,4 & $\begin{array}{l}\text { Using AHP model and measuring } \\
\text { against customised heuristics. }\end{array}$ \\
\hline Qualitative & 3,4 & $\begin{array}{l}\text { Based on questionnaire from those } \\
\text { a part of the relevant demographic. }\end{array}$ \\
\hline Quantitative & 1,4 & $\begin{array}{l}\text { Number of taps taken to complete } \\
\text { task was recorded on video for doc- } \\
\text { umentation purposes and review. }\end{array}$ \\
\hline Quantitative & $1,2,4$ & $\begin{array}{l}\text { Time taken to complete task was } \\
\text { recorded on video for documenta- } \\
\text { tion purposes and review. }\end{array}$ \\
\hline $\begin{array}{l}\text { Qualitative/ } \\
\text { Quantitative }\end{array}$ & 4 & Mixed methods, all of the above. \\
\hline
\end{tabular}

\begin{tabular}{|c|c|}
\hline $\begin{array}{l}\text { Heuristic } \\
\text { Addressed }\end{array}$ & Formulated Question \\
\hline 1 & $\begin{array}{l}\text { Q1 Was there a reasonable loading waiting } \\
\text { time? }\end{array}$ \\
\hline 3 & $\begin{array}{l}\text { Q2 How familiar were you with the terms and } \\
\text { symbols used? }\end{array}$ \\
\hline 2 & $\begin{array}{l}\text { Q3 Was the design suggestive of what you } \\
\text { could do text? }\end{array}$ \\
\hline 6 & Q4 Did you find the given tasks easy to do? \\
\hline 5 & Q5 How consistent were the button sizes? \\
\hline 6 & Q6 How simple was the overall navigation? \\
\hline 9 & $\begin{array}{l}\text { Q7 How aesthetically pleasing is the overall } \\
\text { interface? }\end{array}$ \\
\hline 10 & $\begin{array}{l}\text { Q8 How well designed is the application for } \\
\text { the purpose? }\end{array}$ \\
\hline
\end{tabular}

An experiment applying usability inspection methods to better understand how to engage heuristic evaluation with users in the context of interior design testing UI and UX of MAR applications. This experiment aims to define a method of measuring application effectiveness by:
1. Identifying usability performance limitations

2. Identifying visual and cognitive limitations

3. Identifying the 'effective' and 'ineffective' aspects through a questionnaire completed by participants that are a part of the relevant demographic

4. Validating improvements to inform the last prototype, Prototype C.

\section{Participant Requirements and Demograph- ics}

To standardise the constants of the experiment, it was crucial to select participants who are in an occupation related to the context of the project. This is because the usability of the application must "consider the context in which the system will be used" (Stone et al, 2005).

Here, participants were chosen on the basis that:

- they are employed in one of the below listed roles at PTW Architects Sydney office,

- have adequate knowledge of the use of technological applications, and

- support the means of the research focus, through interest or availability.

There were 15 participants who took part in the study:

Architect (8), Interior Designer (2), Computational Designer (1), Graphic Designer (1), Knowledge Manager (1), Marketing Coordinator (1), and Receptionist (1).

\section{Experiment: Standardising Procedure}

Each interview took between 15 and 30 minutes to complete. Each participant was given the same introductory briefing about the project, and the tasks were given in the same order. By standardising the procedure of each participant interview, it minimises the chance of variance (table 5).
Table 3

Experiment Aims and Methods.

Table 4

Heuristic influences on questionnaire to collect qualitative data. 


\section{Data Collection}

Quantitative data included:

- Tap counts: how many times a participant tapped the touch screen, counting stops when they have achieved the task requirements.

- Time taken: how many seconds the participant took to complete the set task/s, timer begins at first tap on the screen until the last tap that completes the task.

- Ratings using AHP model: the value the participant 'rated' each heuristic (criteria) aspect based on their experiences.

Table 5

Experiment procedure.

Table 6

Anticipated tap count and time taken to complete task.

6 Complete questionnaire and / or further discussion and questions with the researcher Participants can review prototypes on given reference sheet both prototype A and prototype B.

- Preferred prototype: subjective opinion of their preferred prototype.

\section{Anticipated Quantitative Results}

To compare the success of a users' ability to complete tasks at ideal rates, the anticipated tap count and time taken for both prototype $A$ and prototype $B$ have been recorded (table 6). The anticipated rate is the minimum tap count and time taken for a user to complete the task requirements. Anticipated tap counts remain the same in both prototypes due to the same number of steps required to complete the given task.

\section{Results}

Quantitative

Task completion time average (sec): Prototype A: 72.6s, Prototype B: $43.6 \mathrm{~s}$

Tap counts average: Prototype A: 20.93, Prototype B: 15.06

- No participants using prototype A achieved the anticipated rate of 9 tap counts, the minimum was 12 . However, in prototype B, two participants achieved the anticipated rate of 9 tap counts.

No participants using prototype A achieved the anticipated time taken of 34 seconds. While 8 participants using prototype $B$, beat the anticipated time taken of 34 seconds.

- Prototype A's mean tap count was 21 while the mean time taken was $73 \mathrm{~s}$. While in prototype $B$, the mean tap count was 15 and the mean time taken was 44s. A difference of 6 taps and $117 \mathrm{~s}$, possibly due to a learning effect of being introduced to a similar user interface in prototype $A$.

- It was found that participants rated prototype $B$ higher, but they still chose prototype $A$ as the most preferred application. Results varied between all participants, although it provides a gauge of user satisfaction and the applica-
- Questionnaire: the written data collected from questions completed after experiencing

\begin{tabular}{lll}
\hline Prototype & $\begin{array}{l}\text { Anticipated Tap } \\
\text { Counts }\end{array}$ & $\begin{array}{l}\text { Anticipated Time } \\
\text { Taken (s) }\end{array}$ \\
\hline A & 9 & 34 \\
B & 9 & 34 \\
\hline
\end{tabular}

Qualitative data included: 
tion 'effectiveness' within the demographic.

\section{Qualitative}

Popular responses (count): \#1 Button positioning (5), \#2 Button combining (5), \#3 Hints and Suggestions (3), \#4 Levels of detail (2), \#5 Library Customisation (1), \#6 Library sync with suppliers (1), \#7 Hide interface with double tap (1), \#8 Geo reference off option (1), \#9 Map show plan of furniture placement (1), \#10 Add textures (1), \#11 Home page not useful (1), \#12 Bigger buttons (1), and the last response \#13 Smaller buttons (1).

\begin{tabular}{ll}
\hline Time (sec) & Task Description \\
\hline 2 & Brief of project purpose and participant tasks \\
1 & Sign participant information statement and \\
consent form & Prototype A task \\
& Choose a space to view Locate the scene \\
& viewer \\
& Place a chair in the scene \\
& $\begin{array}{l}\text { Prototype B task } \\
\text { Choose a space to view Locate the scene } \\
\text { viewer } \\
\text { Place a chair in the scene } \\
\text { Complete questionnaire and / or further dis- } \\
\text { cussion and questions with the researcher } \\
\text { Participants can review prototypes on given } \\
\text { reference sheet }\end{array}$ \\
&
\end{tabular}

\begin{tabular}{lcccccccccc}
\hline Prototype & \multicolumn{1}{c}{ Usability } & \multicolumn{1}{c}{ Visual and Cognitive } \\
\hline & 1 & 2 & 3 & 4 & 5 & 6 & 7 & 8 & 9 & 10 \\
A & $\bullet$ & 0 & 0 & 0 & $\bullet$ & $\bullet$ & 0 & 0 & 0 & 0 \\
B & $\bullet$ & 0 & 0 & 0 & $\bullet$ & 0 & $\bullet$ & 0 & $\bullet$ & $\bullet$ \\
C & 0 & 0 & 0 & 0 & $\bullet$ & 0 & 0 & 0 & $\bullet$ & $\bullet$ \\
\hline
\end{tabular}

Preferred prototype: Prototype A: 60\%, Prototype B: $40 \%$

- It was found that the 'button positioning' of the order of commands should be swapped, where placeholders appeared first and fur- niture drawer appeared second, as buttons should be in the order a user is expected to use them. 'Hints and suggestions' also a major aspect of improving ease of use. As well as, varying 'levels of detail' would assist with different user experience levels

- Those who preferred prototype A favoured its simplicity and ease of use over its aesthetics. Participants choosing prototype $B$ thought the aesthetics of the UI were more appropriate for the context of the application and that the layout was more user friendly.

\section{Prototype C Improvements}

As a result of the prior investigation, a collection of specific usability and visual and cognitive limitations were highlighted in the experiment, where improvements were implemented in the final prototype, Prototype $C$ (table 7).

We argue that this will results in a more refined prototype which covers the needs and requirements of the demographic (the 15 participants who took part in the study).

\section{Comparison Study: Prototypes EVALUATION}

Upon evaluation, the methodologies played a significant role in identifying limitations of prototypes during the iterative design processes. The experiment found that users responded best to experiences involving visual cues or suggestive gestures to guide them through an application. Also providing users with clear warning and exit messages is also beneficial to troubleshoot implications. Both findings were gathered through observation and evaluation of qualitative data collection.

Qualitative data collection provided more insight into the minds of the intended demographic. Through the integration of heuristics into the questionnaire, it directed the feedback content. The quantitative data collection, aligned with the customised heuristics, provided an insight in the effectiveness of the application. The application effec-
Table 7

Prototype C application improvements derived from experiment results.

Table 8

PTW Interior Design application prototypes A, B and C comparison against customised heuristics using AHP model. 
Figure 4

Prototype $\mathrm{C}$

Interface.
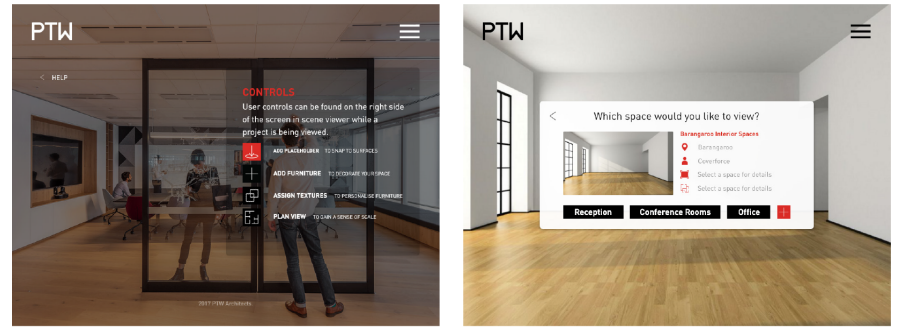

Overview of selected space

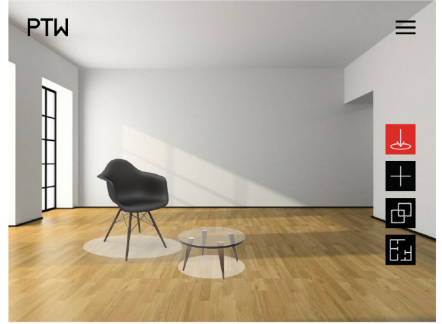

Placina furniture tiveness was examined through a mixed method approach which consisted of: experiments, surveys and inspection methods. It was found that the most appropriate approach for the context of the project was inspection methods, as it directed a non-bias approach for the participants. The research further suggests a method of justifying a suitable list of criteria in the context of interior design. This determined that using heuristics customised for a particular project is more appropriate over generic lists of heuristics.

Applying a modification of existing usability inspection methods, this research consolidates a foundation for developing MAR applications in the context of interior design. As the user trials involved a small sample size of designers (i.e. 15 employees of a renowned design practice), the research could be furthered by testing the prototypes against a larger, and more diversified, sample size (i.e. a clientele demographic). This would provide insight into two different user experience groups, which would highly influence the content of the prototypes and further refine their validity in the iterative design process.

Additionally, a customised set of heuristics was used to evaluate the effectiveness of MAR UI's through user trials with designers. Applying heuristics at the beginning of the design process may provide immediate validation. However, heuristics as an inspection method working in conjunction with user trials is a workflow that proved effective for the aims and time constraints of this project.

\section{CONCLUSION}

In evaluating the design project, the methodologies played a major role in identifying limitations of prototypes during the iterative design processes. The experiment found that users responded best to experiences involving visual cues or suggestive gestures to guide them through an application. Also providing users with clear warning and exit messages is also beneficial to troubleshoot implications.

Both findings were gathered through observation and evaluation of qualitative data collection. Qualitative data collection provided more insight into the minds of the intended demographic. Through the integration of heuristics into the questionnaire, it directed the feedback content. The quantitative data collection aligned with the customised heuristics, provided an insight in the effectiveness of the application. The application effectiveness was examined through a variety mixed method approach which consisted of; experiments, surveys and inspection methods. It was found that the most appropriate approach for the context of the project was mixed methods, as it directed a non-bias approach for the participants.

The paper further suggests a method of justifying a suitable list of criteria in the context of interior design. This determined that using heuristics customised for a particular project is more appropriate over generic lists of heuristics. The processes discussed in this paper can be applied to multiple sce- 
narios as it provides a benchmark for future $\mathrm{UI}$ and UX studies of MAR applications. Therefore, it is evident that the research provides a fundamental understanding of the requirements to achieve an effective UI and UX in MAR applications. Providing an insight for opportunities within AR technologies, utilised as a design communication tool in an interior design context.

\section{ACKNOWLEDGEMENTS}

I would like to take this opportunity to thank Diane Jones, Michael Yip and Tiara Dobbs at PTW Architects for continuous support and direction over the duration Project Lyrebird, the project name for this joint research investigation with Harris Paneras. I would also like to thank the Interior Design and Marketing Team at PTW Architects for their interest and support of our aims and intentions of the project.

Thank you to all the employees who participated in my experimental study. Finally, thank you to Harris Paneras and his contribution to expanding the research scope.

\section{REFERENCES}

Hoo, M.H.H. and Jaafar, A 2013 'An AHP-based approach in the early design evaluation via usability goals', Lecture Notes in Computer Science (including subseries Lecture Notes in Artificial Intelligence and Lecture Notes in Bioinformatics), pp. 694-706

Munzner, T 2009, 'A Nested Model for Visualization Design and Validation.', leee Transactions On Visualization And Computer Graphics, 15(6), p. 921-928

Nielsen, J 1994 'Enhancing the explanatory power of usability heuristics', Proceedings of the SIGCHI Conference on human factors in computing systems, pp. 152-158

Paneras, H, Yip, M, Dobbs, T, Doherty, B, Fabbri, A, Gardner, $\mathrm{N}$ and Haeusler, M. Hank 2018 'Augmented Reality in the Design Process - Using visual effects (VFX) motion tracking techniques to conduct quantification research on the performance of augmented reality', Kepczynska-Walczak, A, Bialkowski, S (eds.), Computing for a better tomorrow - Proceedings of the 36th eCAADe Conference - Volume 2, Lodz University of Technology, Lodz, Poland, 19-21 September 2018, pp. 761-770
Stone, D 2005 'User interface design and evaluation', Elsevier, Amsterdam

Zuk, T, Schlesier, L, Neumann, P, Hancock, S. M and Carpendale, S 2006 'Heuristics for Information Visualization Evaluation', Proceedings of the 2006 AVI Workshop on BEyond Time and Errors: Novel Evaluation Methods for Information Visualization 